\title{
DEM simulations: mixing of dry and wet granular material with different contact angles
}

\author{
Steffen Schmelzle ${ }^{1} \cdot$ Herrmann Nirschl $^{1}$
}

\begin{abstract}
In solid mixing the raw materials typically differ at least in one material property, such as particle size, solid density and wetting properties, which in turn influence particle mobility. For example, smaller particles can percolate through the voids of larger ones under the influence of strain and gravity. This may produce fine particle accumulation at the bottom of the mixing vessel which results in undesired, inhomogeneous final products. When wet particles with different wetting properties need to be mixed, heteroagglomeration may occur as another segregation mechanism. We present a new capillary bridge force model to study segregation in moist cohesive mixing processes using DEM. New analytical equations of best fit are derived by solving the Young-Laplace equation and performing a regression analysis, in order to investigate discontinuous mixing processes of dry and moist materials with different particle sizes and different contact angles. Compared to a dry mixing process, mixing efficiency is improved by the addition of a small amount of liquid. While percolating segregation is reduced, heteroagglomerates occur in the wet mixing process.
\end{abstract}

Keywords Solids mixing $\cdot$ DEM simulation $\cdot$ Liquid bridge model $\cdot$ Segregation $\cdot$ Young-Laplace

\section{Introduction}

\subsection{Problem description}

Solid mixing is one of the main industrial unit operations in mechanical process engineering. Although a variety of mixing principles and mixers are available, segregation effects may occur in different industrial applications, which can result in a reduced product quality. Particle size is the most important factor in particle segregation [1,2]. For example, smaller particles can move through the voids between bigger particles and accumulate at the bottom as a result of gravity. Hence, mixing components that differ greatly in size are more likely to segregate due to percolation than mixing components with smaller size differences [3]. This percolating segregation is less pronounced when smaller particle ranges or powders with large cohesive strength need to be mixed [4].

Steffen Schmelzle

steffen.schmelzle@kit.edu

1 Institute for Mechanical Process Engineering and Mechanics, Karlsruhe Institute of Technology, Strasse am Forum 8, 76131 Karlsruhe, Germany
By adding small amounts of liquid, the differing motions of the mixing components are compensated by interparticle liquid bridge forces. Smaller particles can adhere to bigger particles, resulting in more homogeneous products [1]. However, liquid bridge forces depend on the liquid properties [5] and on the particle properties like size or wetting behaviour [6]. This in turn may improve or deteriorate the mixing result depending on whether emerging agglomerates may be broken or not [7]. For example, if components with different wetting behaviour like limestone powder and non-expanded polystyrene need to be mixed, water accumulates selectively in the limestone, such that heteroagglomerates are formed [8]. Agglomerates consisting of mainly one component are an undesirable inhomogeneity. Accordingly, it cannot generally be assumed that the addition of liquid minimizes segregation. This has to be assessed on a case by case basis depending on the feed and product characteristics.

This research is aimed at understanding segregation like percolation and heteroagglomeration in more detail using numerical methods. In particular, it is observed how liquid and especially different wetting properties of the mixing components influence segregation. For this purpose, the liquid bridge model in the Discrete Element Method (DEM) is developed further for more accurate numerical simulations. 


\subsection{State of modelling moist particulate solids in DEM}

The DEM is a numerical method to investigate complex movement of bulk materials, which allows optimizing forecasts for particulate-related processes. Based on the ideas of Cundall and Strack [9], the particle is represented as a regular sphere with mass $m_{i}$ that is accelerated by field and contact forces according to Newton's law of motion (Eq. 1):

$m_{i} \frac{d \vec{u}_{i}}{d t}=m_{i} \vec{g}+\sum_{j=1}^{l} \vec{F}_{c, i j}$

Subsequent integration over a short time step $d t$ with an explicit finite difference method leads to the velocities $\vec{u}_{i}$ and the positions of each particle $i$. In the case of solid mixing, the forces acting on each particle are composed of the gravitational force $m_{i} \vec{g}$ and contact forces $\vec{F}_{c n, i j}$, the latter resulting from particle-particle and particle-wall collisions. The contact force between element $i$ and $j$ is modeled as a spring and damping system, composed of an elastic $\vec{F}_{e l, i j}$ and a dissipative term $\vec{F}_{d i s, i j}$. The elastic term defines the kinetic energy storage during a collision as a function of the spring constant $k_{n}$, the normal vector $\vec{n}_{i j}$, and the virtual overlap $\delta_{n}$. The damping term describes kinetic energy dissipation depending on the damper constant $\eta_{n}$ and the relative speed $\vec{u}_{r e l, i j}^{n}$ between the particles $i$ and $j$ (Eq. 2):

$$
\vec{F}_{c n, i j}=\vec{F}_{\text {el,ij }}+\vec{F}_{\text {dis }, i j}=-\underbrace{k_{n} \delta^{1,5} \vec{n}_{i j}}_{\text {spring term }}-\underbrace{\eta_{n} \vec{u}_{r e l, i j}^{n}}_{\text {damping term }}
$$

The DEM simulations are performed with EDEM 2.7, a commercial software in which the contact model according to Tsuji et al. [10] is used.

In moist mixing processes with small amounts of liquid, stable, rotationally symmetric liquid bridges are formed, which produce an attractive force due to capillary pressure and surface tension. When simulating moist particle systems, the attractive liquid bridge force $\vec{F}_{b, i j}$ has to be additionally implemented in Newton's law of motion (Eq. 1)). Various approaches have been developed to investigate the liquid bridge force at constant liquid volumes. All these approaches have in common that the liquid bridge is stable up to a critical particle separation distance at which the bridge ruptures. Mikami et al. [11] were among the first authors to develop a regression expression for the liquid bridge force and the rupture distance based on the numerical solution of the Young-Laplace equation. Their model has been used successfully to perform a DEM simulation of a fluidized bed. Remy et al. [12] transferred this model to cohesive batch mixing processes and validated their numerical findings experimentally using particle image velocimetry. This work provided interesting insights into mixing kinetics and local agglomeration in a bladed mixer at different moisture contents. However, this model is restricted to the contact interactions of monodisperse spheres. Soulie et al. [13] expanded the dimensionless liquid bridge force model to polydisperse granular materials, which was used by Anand et al. [14] to investigate the segregation of cohesive particle systems during discharge from a drain funnel. When transferred to mixing processes, this model could be used to describe the contact mechanisms between particles consisting of the same material. However in mixing processes there are often two materials that differ, for example, in solid density and wetting properties.

The complete mathematical description of liquid bridges, a problem already known for over two centuries, is still not solved, as obvious from the work of Lian and Seville [6]. On the basis of many numerical calculations, they presented new explicit analytical equations to describe the liquid bridge force, the liquid volume and the rupture distance. Their equations cover a wide field of different particle sizes and represent a very good approximation for the description of a polydisperse bulk material with a broad size distribution. These closed-form equations would be ideal, if variable wetting characteristics were included as well. Butt and Kappl [15] extended the Derjaguin [16] approximation with the simplification of a mean cosine of the contact angles, in order to take into account both different particle radii and different contact angles. This toroidal approximation is based on the assumption of a very small half filling angle and is consequently limited to small liquid bridge volumes only.

\subsection{Objective}

In summary, the implementation of liquid bridges in DEM and the numerical simulation of moist, cohesive bulk materials can be accomplished already with simplifying assumptions. However, the existing liquid bridge models are often limited to only one group of particles or just describe simplistic monodisperse systems. These models offer only limited applicability when dry and moist particles with different properties, such as particle size and wetting behaviour, have to be simulated. For this reason, we developed new analytical equations of best fit for the liquid bridge force, the rupture distance, and the liquid redistribution. These equations are used subsequently to investigate the mixing process of dry and moist bulk materials.

\section{Mixing technology}

\subsection{Batch mixer and simulation settings}

The geometry used for the DEM simulations was a computeraided design (CAD) model (Fig. 1a) of a pharmaceutical 

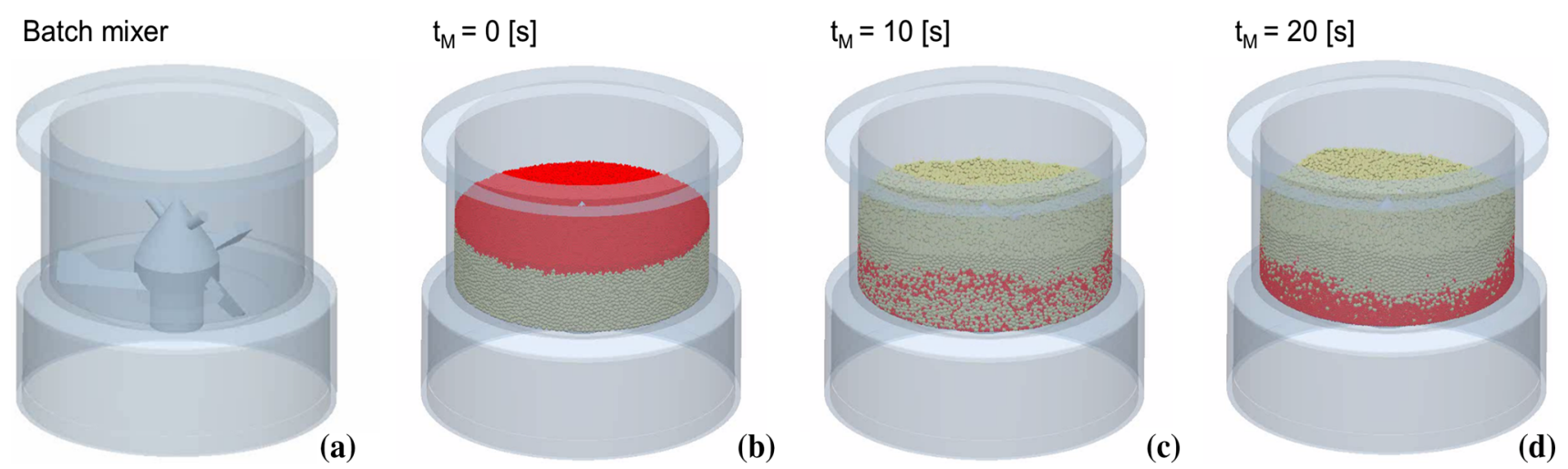

Fig. 1 3D Visualization of the discontinuous mixer (a) and screenshots of the initial condition (b) and different mixing times $t_{M}(\mathbf{c})$, (d) showing the percolating segregation. The rotational speed is set to $36 \mathrm{rpm}$. Figure 1 corresponds to the gray dashed line in Fig. 2

Table 1 Material properties used in the simulations

\begin{tabular}{lll}
\hline Properties & Value & Unit \\
\hline Filler particle $r_{i}$ & 0.92 & {$[\mathrm{~mm}]$} \\
Additive particle $r_{j}$ & 0.46 & {$[\mathrm{~mm}]$} \\
Particle density $\rho_{P}$ & 2.500 & {$\left[\mathrm{~kg} / \mathrm{m}^{3}\right]$} \\
Wall density $\rho_{\text {steel }}$ & 7800 & {$\left[\mathrm{~kg} / \mathrm{m}^{3}\right]$} \\
Shear modulus $G_{p}$ of particles & 216 & {$[\mathrm{MPa}]$} \\
Shear modulus $G_{\text {steel }}$ of steel (mixer) & 8000 & {$[\mathrm{MPa}]$} \\
Coefficient of restitution $e_{p}$, particle to & 0.6 & {$[-]$} \\
$\quad$ particle & & \\
Coefficient of restitution $e_{s t e e l}$, particle to & 0.7 & {$[-]$} \\
$\quad$ particle & & \\
Poisson's ratio of particles $v_{P}$ & 0.25 & {$[-]$} \\
Poisson's ratio of steel $v_{\text {steel }}$ & 0.3 & {$[-]$} \\
Static friction of particles on glass $\mu_{s, p}$ & 0.5 & {$[-]$} \\
Static friction of particles on steel $\mu_{s, \text { steel }}$ & 0.32 & {$[-]$} \\
Rolling friction of particles on glass $\mu_{r, p}$ & 0.005 & {$[-]$} \\
Rolling friction of particles on steel & 0.02 & {$[-]$} \\
$\quad \mu_{r, \text { steel }}$ & & \\
\hline
\end{tabular}

mixer. At the bottom of the mixing vessel, a three-blade impeller rotates at different rotational speeds. The mixing vessel is cylindrical with a diameter of $111.4 \mathrm{~mm}$ and a height of $70.3 \mathrm{~mm}$, resulting in a volume of approximately $685 \mathrm{ml}$ (without the impeller). For our study, 50,000 particles per particle fraction were created in all simulations with a random distribution algorithm leading up to 100,000 particles in total. The red additive particle fraction was generated after the gray filler fraction resulting in the initial state shown in Fig. 1b. The filler particles are two times bigger than the additive component.

The Hertz-Mindlin contact model parameters were selected in conformity with other works $[12,17]$ in order to describe glass beads. A compilation of the mechanical and physical properties used in the simulation is given in Table 1.

\subsection{Evaluation of mixing processes}

In order to evaluate the quality of mixing, the composition of $N$ virtual samples need to be taken and analyzed. The composition of each sample $c_{i}$ fluctuates around an average value $\bar{c}$. As the number of particles is defined in the pre-processing step of the simulation, it is not necessary to calculate the average value. The same number of filler and additive particles leads to an average number composition of $\bar{c}=0.5$. In order to avoid compensation of positive and negative deviations $\left(c_{i}(t)-\bar{c}\right)$, the square of the differences $\left(c_{i}(t)-\bar{c}\right)^{2}$ is used to calculate the empirical variance $s(t)^{2}$ (Eq. 3). Based on the empirical variance, the well-known [18] and often used relative standard deviation $R S D$ is determined as a mixing index according to Eq. (4):

$$
\begin{aligned}
& s(t)^{2}=\frac{1}{N} \sum_{i=1}^{N}\left(c_{i}(t)-\bar{c}\right)^{2}, \\
& R S D=\frac{\sqrt{s(t)^{2}}}{\bar{c}} .
\end{aligned}
$$

It is impossible to reach ideal homogeneity $R S D_{i d}=0$, because random particle motions always occur in solid mixing processes. The best achievable quality of mixing is characterized by the uniform random mixture $R S D_{r}$, which corresponds to the smallest detectable relative standard deviation. In the case of constant solid density, this limiting value can be estimated with the sample mass $m_{P}$ and the mass of a single additive particle $m_{A}$ according to Eq. (5):

$R S D_{r}=\frac{\sqrt{s_{r}^{2}}}{\bar{c}}=\frac{\sqrt{\bar{c}(1-\bar{c}) \cdot \frac{m_{A}}{m_{P}}}}{\bar{c}}$.

The mixing efficiencies shown in Fig. 2 are determined by taking 16 randomly distributed sampling locations, which are analyzed every $0.2 \mathrm{~s}$. With an average sampling mass of 


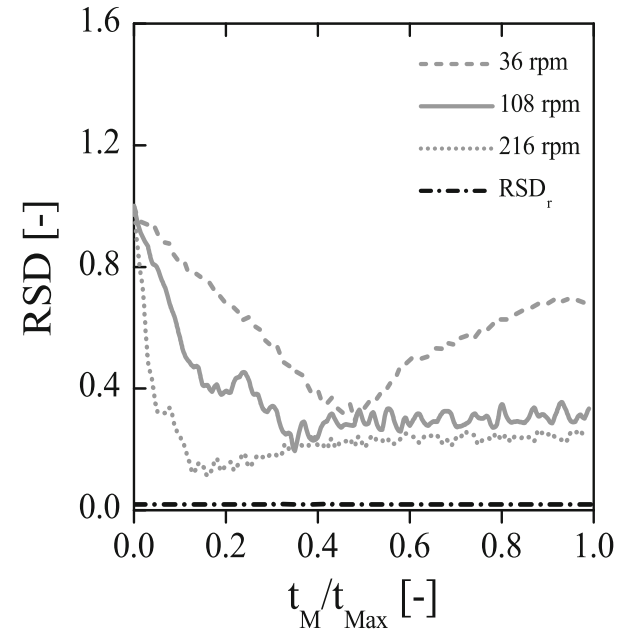

Fig. 2 Mixing efficiencies determined for different rotational speeds (particle size ratio $\Psi=r_{i} / r_{j}=2.0$, simulation time $t_{\operatorname{Max}}=20 \mathrm{~s}$ )

$m_{p}=3.0 \mathrm{~g}$, approximately 600 particles are detected per sample.

In the completely segregated initial state at the mixing time $t_{M}=0$ (see Fig. 1a), the additive particles are located on top of the coarse filler particles, which is specified by the relative standard deviation $R S D_{t_{M}=0}=1$. When starting the mixing process at 36 revolutions per minute, homogeneity improves with progressing mixing time until the best homogeneity is reached after $10 \mathrm{~s}$ (see also Fig. 1c). With further mixing homogeneity declines again as smaller particles move through the voids of the coarse particles and begin to accumulate at the bottom of the vessel (Fig. 1d). After $20 \mathrm{~s}$ of mixing most of the additive particles are located at the bottom, which is reflected by an increased $R S D$. This percolating segregation mechanism still exists at higher rotational speeds. Due to the higher energy input, more dispersive particle motions occur resulting in less pronounced convective transport in the direction of the gravity field. Consequently, an increase in speed leads to better temporary and final homogeneities. However, the best possible quality of mixing $\left(R S D_{r}\right)$ cannot be reached in all three conditions.

As mentioned in Sect. 1, the addition of liquid influences this segregation effect. Due to cohesive forces, the relative particle mobility can be reduced and thus, homogeneity may be improved. Eventually the addition of liquid may cause a change of segregation mechanism towards heteroagglomeration, due to different wetting characteristics between the various types of particles. In order to investigate the change of segregation mechanisms in DEM simulations, an extension of the existing liquid bridges has to be carried out.

\section{Liquid bridge model}

Depending on the moisture content, wet particle systems can exist in either the pendular liquid bridge state, the funicular state, or the capillary state. Research reported here covers moist mixing processes with small amounts of liquid, which can be sufficiently described by liquid bridge networks. When a small amount of liquid is introduced between two contacting solid particles, a stable, rotationally symmetric meniscus is formed (Fig. 3) which can produce an attractive liquid bridge force due to capillary pressure $\Delta p$ and surface tension $\gamma$. The relationship between capillary pressure and surface tension between the two fluid phases is given by the elliptic Young-Laplace differential equation in dimensionless form (Eq. 6),

$$
\begin{aligned}
2 H^{*}= & \frac{\Delta p \cdot r_{i j}}{\gamma}=\frac{\ddot{Y}(X)}{\left(1+\dot{Y}(X)^{2}\right)^{\frac{3}{2}}} \\
& -\frac{1}{Y(X)\left(1+\dot{Y}(X)^{2}\right)^{1 / 2}},
\end{aligned}
$$

where $H^{*}$ is the dimensionless mean curvature and $Y$ and $X$ are the dimensionless coordinates with respect to the harmonic radius $r_{i j}$ given by Eq. (7):

$r_{i j}=\frac{2 r_{i} r_{j}}{r_{i}+r_{j}}, \quad Y=\frac{y}{r_{i j}}, \quad X=\frac{x}{r_{i j}}$.

\subsection{Numerical solution of the Young-Laplace equation}

As a first approximation, Fisher [19] presented the liquid bridge profile as a toroid (constant meridional radius) which leads to a capillary bridge force that characterizes liquid bridges with very small volumes only. In order to determine the exact liquid bridge profile for every possible bridge geometry and hence, to derive the exact liquid bridge force, numerical methods have to be used. Hotta et al. [20] were among the first to solve the Young-Laplace equation with numerical methods for a sphere-plate application. Schubert [21] first published solutions for the sphere-sphere application without explicitly explaining the numerical approach, so that the often cited work of Lian et al. [22] serves as a basis for the numerical calculation of the liquid bridge force as a function of the separation distance. They assume a constant liquid volume while separating two solid particles up to a critical rupture distance $s_{B}$. Beyond the critical rupture distance, no stable liquid bridge profile can be observed. It is found that the rupture distance for capillary bridges between same grain particles depends on the cubic root of the liquid volume $v$. For this reason, the solution of the Young-Laplace equation was approximated by a truncated Taylor series (Eq. 8), which describes the meridian liquid bridge profile $Y(X)$ : 


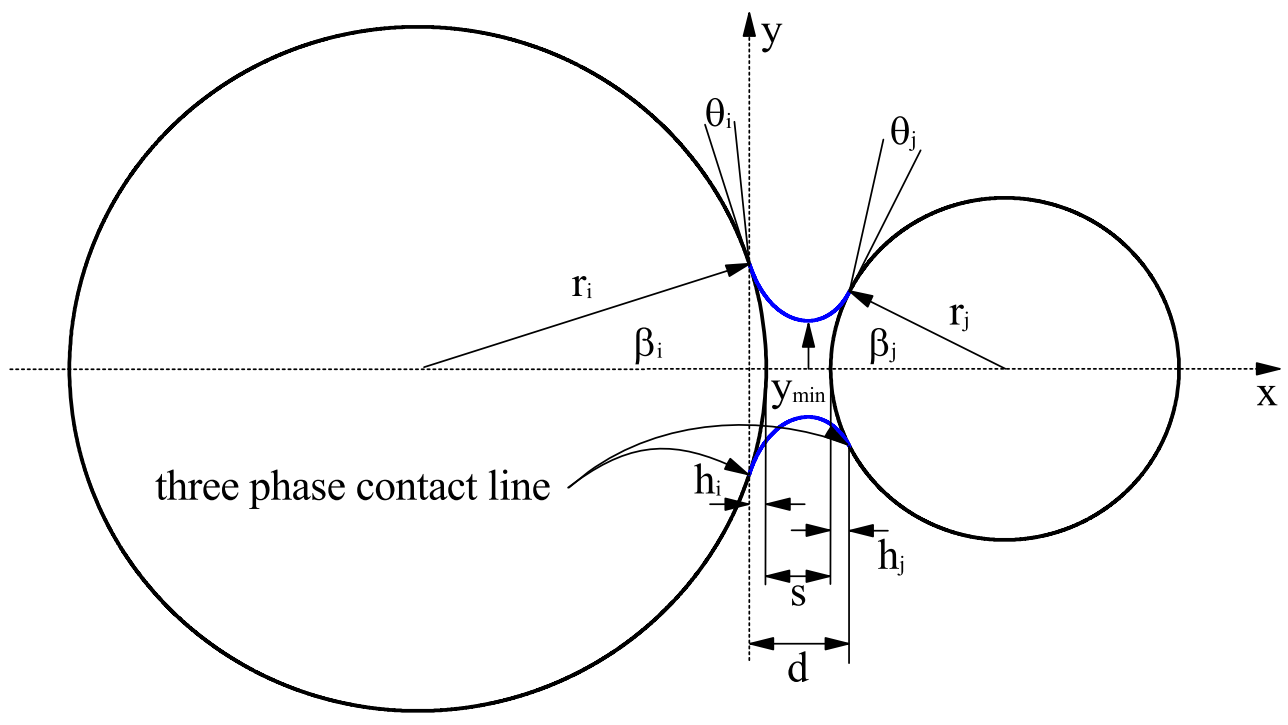

Fig. 3 Sketch of a liquid bridge between two unequal spheres with different contact angles. By definition the three phase contact line of particle $i$ is set to $x=0$

$$
\begin{aligned}
Y_{i+1} & =Y_{i}+\left(X_{i+1}-X_{i}\right) \dot{Y}_{i}+\frac{1}{2}\left(X_{i+1}-X_{i}\right)^{2} \ddot{Y}_{i} \\
i & =0,1,2, \ldots
\end{aligned}
$$

A subsequent substitution $Q=\ddot{Y}^{2}+1$ transforms Eq. (6) into the Bernoulli Eq. (9), whose integration constant $C$ is determined by using the boundary conditions at $X=0$ and $X=D$ (Eqs. 10 and 11):

$\frac{Y}{\left(1+\dot{Y}^{2}\right)^{0,5}}+H^{*} Y^{2}=C$

$Y(0)=R_{i} \sin \left(\beta_{i}\right), \quad \dot{Y}(0)=-\cot \left(\beta_{i}+\theta_{i}\right)$,

$Y(D)=R_{j} \sin \left(\beta_{j}\right), \quad \dot{Y}(D)=\cot \left(\beta_{j}+\theta_{j}\right)$,

where $R_{i}=r_{i} / r_{i j}$ and $R_{j}=r_{j} / r_{i j}$ are the dimensionless particle radii. As boundary condition of Eq. (9) the half filling angle $\beta_{j}$ on particle $j$ has to match the predefined half filling angle at the three phase contact line. In addition, the gradient of the curvature and the gradient of particle $j$ in the contact line must differ around the contact angles $\theta_{j}$. For a contact angle of zero, both gradients (particle and bridge profile) must be the same. The integration constant, which correlates the boundary conditions between particles $i$ and $j$, is calculated from Eq. (12):

$$
\begin{aligned}
& \frac{Y(0)}{\left(1+\dot{Y}(0)^{2}\right)^{0,5}}+H^{*} Y(0)^{2}=\frac{Y(D)}{\left(1+\dot{Y}(D)^{2}\right)^{0,5}} \\
& +H^{*} Y(D)^{2}=C .
\end{aligned}
$$

The liquid bridge profile (Eq. 9) was calculated with the following numerical procedure. The particle radii and contact angles were predefined and the dimensionless particle distance $S=\mathrm{s} / r_{i j}$ was initially set to zero. Subsequently, a value of $Y(0)$ was selected that can be used to approximately describe the dimensionless liquid bridge volume $V=v / r_{i j}^{3}$, which is calculated by integration over the interval $x=0$ and $x=d$ according to Eq. (13):

$$
\begin{aligned}
V= & \frac{\pi}{r_{i j}^{3}} \int_{0}^{d} y^{2}(x) d x \\
& -\frac{\pi}{6}\left[3 y^{2}(0) h_{i}+h_{i}^{3}+3 y^{2}(d) h_{j}+h_{j}^{3}\right],
\end{aligned}
$$

where $h_{i}$ and $h_{j}$ are the spherical cap heights of each particle that are calculated by simple geometric relationships and $d$ is the distance between both three-phase contact lines (see Fig. 3). The mean curvature $H^{*}$ is not known and is calculated via a Newton iteration scheme to reach an error smaller than $10^{-6}$ in the contact angle of particle $j$. At this time, if the liquid bridge volume does not meet the predefined value, a correction of $Y(0)$ is made using an interval halving iteration method. This procedure is repeated until an error smaller than $10^{-6}$ is reached in the liquid bridge volume as well as in the contact angle of particle $j$. The discretization of $\Delta X$ is set small enough to guarantee this error value. Once a solution is found for the initial condition, the separation distance is increased in order to calculate the liquid bridge profile $Y(x)$ for a constant liquid bridge volume. The increase of the separation distance cannot be carried out infinitely (Fig. 4). At a critical distance, no further solution exists for the corresponding liquid bridge volume.

As well as this stable solution, a second physically unstable solution exists that also meets the criteria of the boundary conditions (Eqs. 10 and 11), as observed in an earlier numerical study [22]. The criterion when both solutions converge 
Fig. 4 Numerical solution of the Young-Laplace equation as a function of the dimensionless separation distance $S$ for a set of different volumes $v\left[\mathrm{~mm}^{3}\right]$ in terms of the dimensionless neck height $Y_{\min }$, the boundary condition $Y(0)$ which correlates to the half filling angle $\beta_{i}$, the dimensionless mean curvature $H^{*}$, and the dimensionless liquid bridge force $F^{*}$ $\left(r_{i}=2 \mathrm{~mm} ; r_{j}=1 \mathrm{~mm}\right.$ and $\theta_{i}=\theta_{j}=0^{\circ}$ )
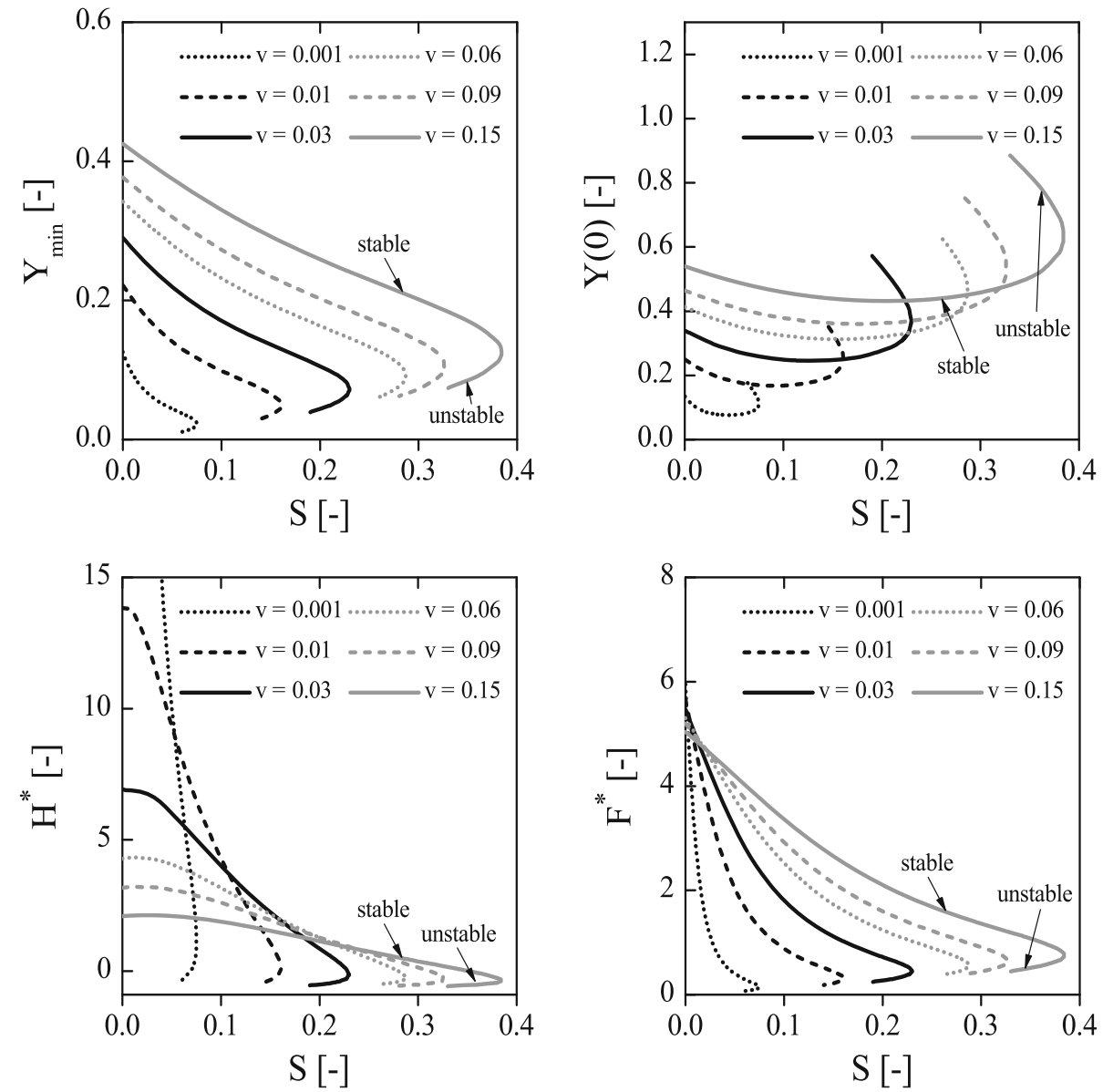

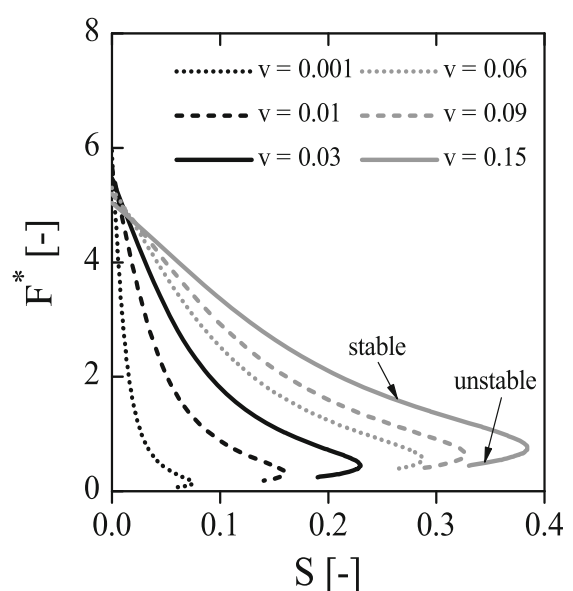

characterizes the critical rupture distance $S_{B}$. Due to the surface tension, the liquid bridge tends to the state with minimized interfacial energy, which is described by the stable solution.

The dimensionless liquid bridge force $F^{*}$ acting between two solid particles is composed of the forces arising from surface tension and capillary pressure. With the calculated liquid bridge profile, the dimensionless bridge force $F^{*}$ can be obtained at the bridge neck height $Y_{\min }$ or at the threephase contact line using Eqs. (14a) or (14b). As the capillary pressure is constant over the local coordinate $X$, both equations lead to the same result:

$$
\begin{aligned}
F^{*}= & \frac{F}{\gamma r_{i j}}=2 \pi Y_{\min }\left[1+H^{*} Y_{\min }\right], \\
F^{*}= & \frac{F}{\gamma r_{i j}}=2 \pi Y(X)\left[1 /\left(1+\dot{Y}(X)^{2}\right)^{0,5}\right. \\
& \left.+H^{*} Y(X)\right] \quad X=0 \quad \text { or } \quad X=D .
\end{aligned}
$$

Figure 4 displays plots of the numerical solution for a set of different volumes. As shown in Eq. (14a), the force decreases with decreasing neck height and mean curvature, while a minimum is observed in the height of the threephase contact line of particle $i$, which correlates to the half filling angle $\beta_{i}$. Additionally, the force decreases monotonically with increasing separation distance for all volumes, while larger liquid volumes remain stable over a bigger range. Willett et al. [23] used this numerical procedure to show a very good agreement between experimentally measured and numerically calculated capillary forces. Lian and Seville [6] recently presented new closed-form equations obtained by the numerical solution of Young-Laplace's equation. Their new equations characterize a bulk material with a broad size distribution. We used the numerical method to calculate the liquid bridge forces between two particles of different materials. Accordingly, the contact angle of particle $j$ has been varied, while a size difference exists between particle $i$ and $j$. This results in variable wetting behaviours of the additive material (red particles) in the DEM simulations.

\subsection{Regression analysis}

Due to the high computational effort required for DEM simulations, the Young-Laplace equation cannot be solved for each contact event. Hence, we performed a regression analysis in order to implement the liquid bridge force as an additional empirical equation in Newton's law of motion (Eq. 1). 
Fig. 5 Dimensionless liquid bridge force as a function of the separation distance $S$ for a contact angle of $\theta_{j}=0^{\circ}$ (a) and $\theta_{j}=60^{\circ}$ (b). The green curvatures represent the equation of best fit (Eq. 15)
Fig. 6 a Rupture distance $S_{B}$ as a function of the dimensionless liquid bridge volumes $V$ and different contact angles of the additive material (particle $j$ ). b Transfer ratio $T F_{i}=V_{i} / V$ calculated from the bridge profile at rupture distance for the same set of liquid bridge volumes and contact angles. The green curves represent the equations of best fit Eqs. (16) and (17)
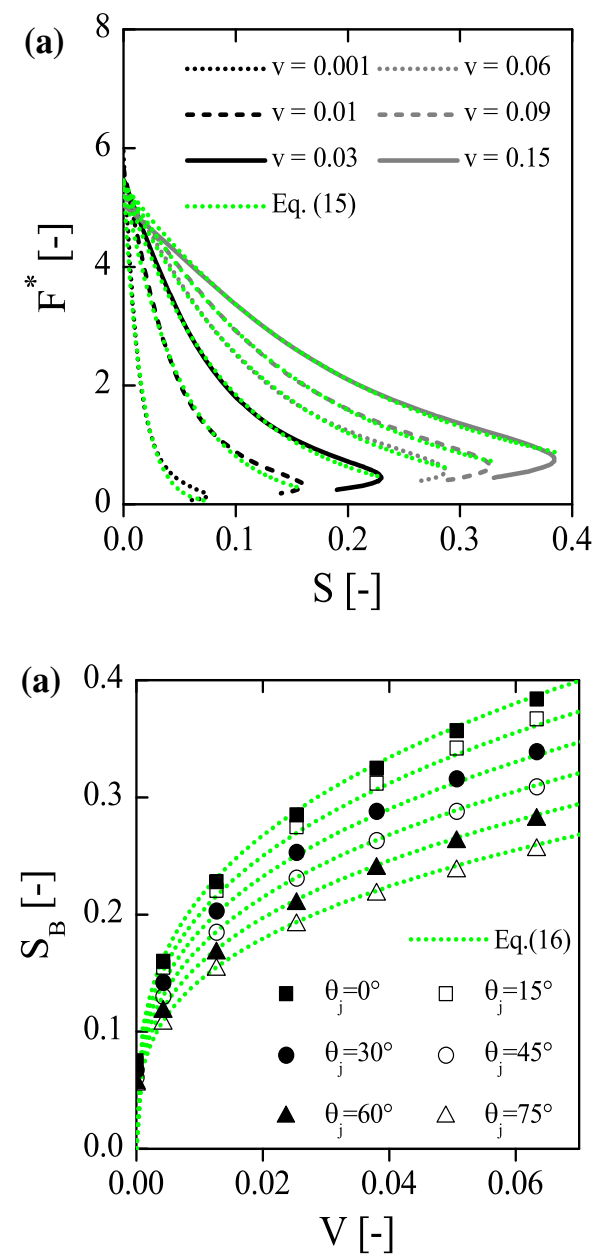
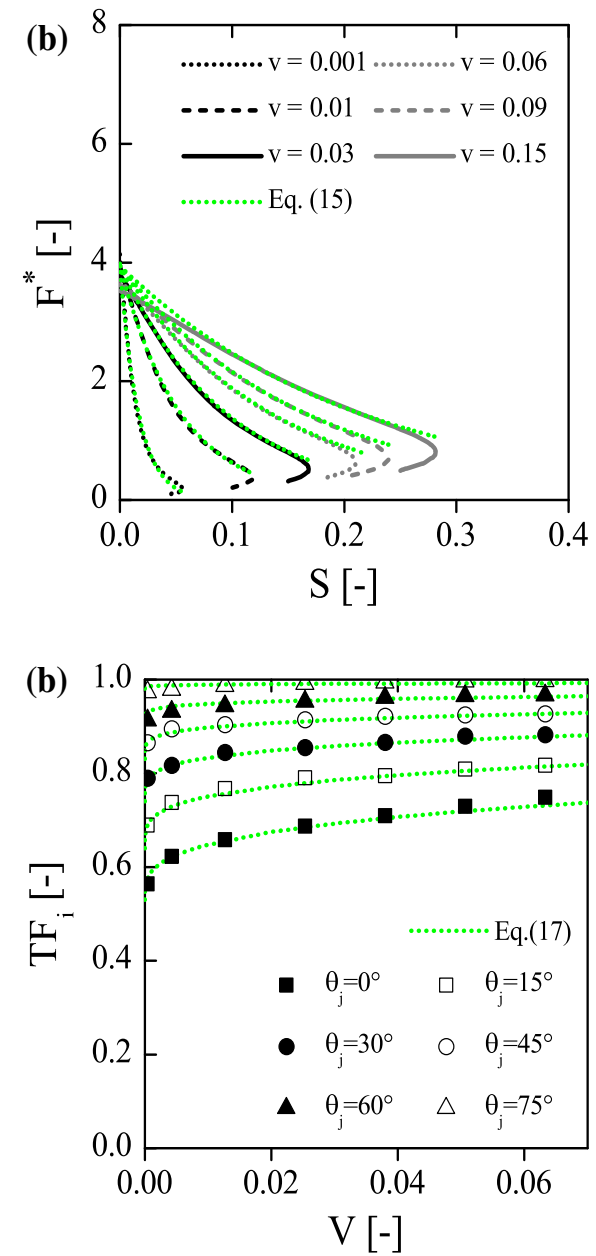

Figure 5 shows the numerical results and the equation of best fit (Eq. 15) of the dimensionless liquid bridge force for a contact angle of $\theta_{j}=0^{\circ}$ (Fig. 5a) and $\theta_{j}=60^{\circ}$ (Fig. 5b). Comparison reveals a reduction of the liquid bridge force as the contact angle increases. Moreover, the empirical equation found is in very good agreement with the calculated results.

$$
\begin{aligned}
F^{*}= & \exp (A+B S), \\
A= & \left(-0.2 \theta_{j}^{2}-0.09 \theta_{j}+1.68\right) \\
& \cdot V^{\left(-0.0005 \theta_{j}^{2}+0.0005 \theta_{j}-0.0013\right)}, \\
B= & -0.1-1.1 / V^{\left(0.52-0.005 \theta_{j}\right)} .
\end{aligned}
$$

The numerical solutions also give information about the rupture distances and the transfer ratios. Regarding the rupture distance (Fig. 6a), a reduction is found with decreasing liquid volume. Similar to the case of equally sized particles the rupture distance scales with the volume raised to the power of approximately one third Eq. (16):

$S_{B}=\left(0.936-0.235 \theta_{j}\right) \cdot V^{0.32}$.

In the case of liquid bridge rupture, it is natural to assume the rupture event to happen at the thinnest part of the liq- uid bridge at $Y_{\min }$ [24]. Thus, the liquid bridge volume separates into two different amounts $V_{i}$ and $V_{j}$ remaining with the single particles. For spheres of the same size with the same contact angle, the simple case is that both particles gain the same amount of liquid, characterized by $T F_{i}=V_{i} / V=V_{i} /\left(V_{i}+V_{j}\right)=0.5$. When differently sized particles with variable contact angles are considered, the transfer ratio has to be calculated by integrating the liquid bridge profile and subtracting the corresponding spherical cap volume. In the case shown here (Fig. 6b) a transfer ratio higher than 0.5 is observed. This indicates a larger amount of liquid remaining with the bigger particle. Secondly, almost no liquid is transferred to small particles with high contact angles. This results in particularly poor wetting properties. Again, the set of data is described very well by means of a fit Eq. (17), which ensures transfer ratios between 0 and 1:

$$
\begin{aligned}
T F_{i} & =C+(1-C) \cdot\left(1-\frac{1}{(1+V)}\right)^{D}, \\
C & =-0.073 \theta_{j}^{2}+0.44 \theta_{j}+0.53 \\
D & =0.076 \theta_{j}^{2}-0.186 \theta_{j}+0.3 .
\end{aligned}
$$


Table 2 Applicability of different liquid bridge models

\begin{tabular}{|c|c|c|c|c|}
\hline References & Applicability & Bridge force $F^{*}$ & Rupture distance $S_{B}$ & Transfer ratio $T F_{i}$ \\
\hline Lian et al. [22] & Same spheres & - & $S_{B}=(\theta ; V)$ & - \\
\hline Mikami et al. [11] & Same spheres & $F^{*}=(\theta ; V ; S)$ & $S_{B}=(\theta ; V)$ & $50 / 50 \%$ \\
\hline Willett et al. [23] & Polydisperse material & $F^{*}=(\theta ; V ; S)$ & $S_{B}=\left(r_{1} ; r_{2} ; \theta ; V\right)$ & - \\
\hline Soulie et al. [13] & Polydisperse material & $F^{*}=\left(r_{i} ; r_{j} ; \theta ; V ; S\right)$ & - & - \\
\hline Butt and Kappl [15] & $\begin{array}{l}\text { Approximation (valid for small } \\
\text { volumes) }\end{array}$ & $F^{*}=\left(r_{i} ; r_{j} ; \theta_{i} ; \theta_{j} ; V ; S\right)$ & - & - \\
\hline Lian and Seville [6] & Polydisperse material & $F^{*}=\left(r_{i} ; r_{j} ; \theta ; V ; S\right)$ & $S_{B}=\left(r_{1} ; r_{2} ; \theta ; V^{*}\right)$ & - \\
\hline This work & $\begin{array}{l}\text { Different materials with fixed } \\
\text { particle size ratio }\end{array}$ & $F^{*}=\left(\theta_{j} ; V ; S\right)$ & $S_{B}=\left(\theta_{j} ; V\right)$ & $T F_{i}=\left(\theta_{j} ; V\right)$ \\
\hline
\end{tabular}

\subsection{Comparison of liquid bridge models}

Table 2 shows a comparison of liquid bridge models which have been applied in DEM simulations. All these models have in common that the unknown liquid bridge force can be calculated as function of the liquid bridge volume $V$ and the particle separation distance $S$. The difference between these models is the applicability. Lian et al. [22] and Mikami et al. [11] focused on same spheres, Willett et al. [23], Soulie et al. [13] and Lian and Seville [6] derived expressions for polydisperse materials.

The model presented in this work is valid for the contact interaction between different materials, with the restriction of a fixed particle size ratio of two. The contact angles of both particles are the same in the existing models. In this work a variable contact angle of the smaller particle can be used, while a contact angle of zero is assigned to the bigger particle. In comparison to previously published relationships (e.g. [7] and [17]) this leads to a lower range of bridge stability, when the contact angle $\theta_{j}$ is increased (Fig. 6a). This is a physically meaningful result, explained by the simultaneous size and contact angle difference of additive and filler particle. If the liquid transfer between the bigger filler and smaller additive particle needs to be modelled, it would be an incorrect simplification to assume an equal redistribution to both contacting particles, which underlines Eq. (17) as a crucial aspect.

Willett et al. [23] measured capillary forces experimentally for perfect wetting bridges between sapphire spheres (radii of 2.381 and $1.191 \mathrm{~mm}$ ) and $127.8 \mathrm{nl}$ of dimethylsiloxane as a liquid $(\gamma=20.6 \mathrm{mN} / \mathrm{m})$. Their experiment showed a very good agreement with the numerical solution of the Young-Laplace equation and is covered by both our model and the other models (Fig. 7). All model equations describe the case with ideal wetting $\left(\theta_{i}=\theta_{j}=0^{\circ}\right)$ very precisely. The slight overestimation of the Derjaguin approximation [16] at zero separation and large liquid bridge volumes has already been reported by Pitois et al. [25] and has been made more accurate by the recently published closed-form equa-

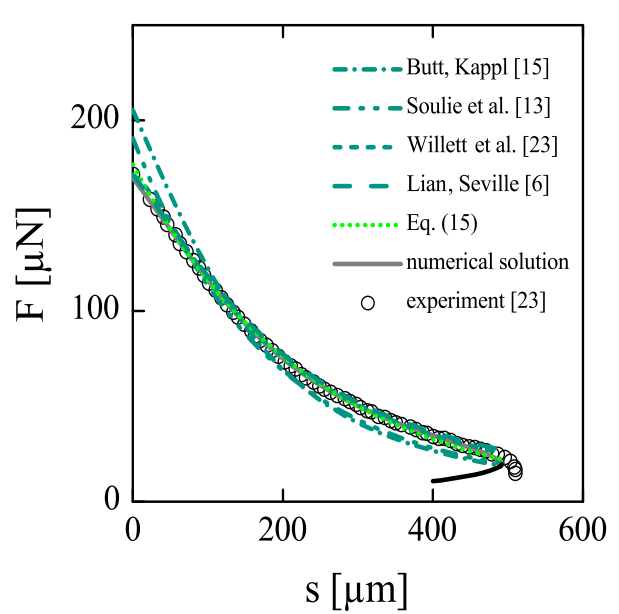

Fig. 7 Comparison of different model equations and the experimental data obtained by Willett et al. [23] for wetting bridges between sapphire spheres. Capillary bridge force $F$ as a function of the separation distance $s$

tion [6]. Our model equation is not intended to improve the existing models but to extend the range of capillary bridge force, rupture distance and transfer ratio calculation as shown in Table 2. The contact interaction between a filler and an additive particle cannot be described satisfactorily in DEM simulations without this model extension. The validation of the new capillary bridge model for particle pairs with different diameters and different contact angles is still an open issue. Here, more experimental data for such configurations is needed.

\subsection{Implementation}

The equation of best fit for the liquid bridge force is implemented as an additional term in Newton's law of motion. However, Eq. (15) cannot be used for every contact event. Depending on the contact partners, three cases have to be distinguished. The liquid bridge force and rupture distance model proposed by Mikami et al. [11] is applied, when a same grain $(i-i)$ or a sphere-wall $(i-w a l l)$ contact occurs. Con- 
(a)

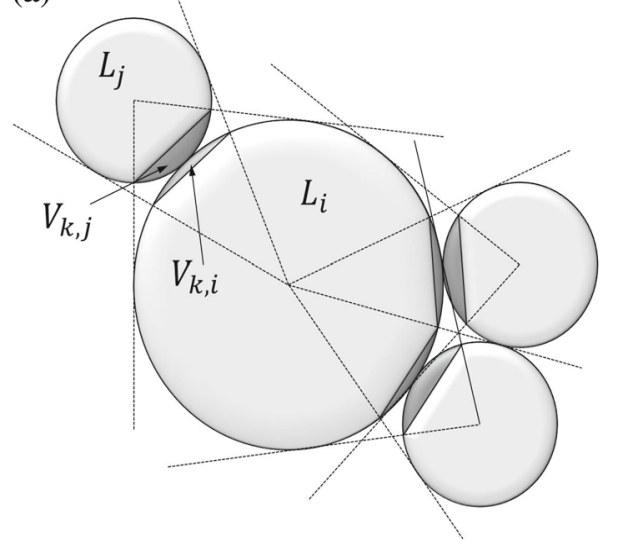

Fig. 8 a Spherical cap model according to Shi and McCarthy [24]. The liquid that is covered by the dark grey caps will contribute to the liquid bridges. The light grey surface displays liquid that is still available for additional liquid bridges, $\mathbf{b}$ illustration of the contact radius

sequently, our model (Eq. 15) is applied to the contact event of the different mixing components $(i-j)$ only.

A liquid bridge is formed instantly, when the physical radii of the particles overlap each other. The same applies to the formation of a liquid bridge between a sphere and the wall. In the event of liquid bridge formation, the volume of the liquid bridge is calculated according to the spherical cap model [24]. In the schematic model shown in Fig. 8a, the liquid content $\left(L_{i}\right)$ on particle $i$ will contribute to three liquid bridges. The liquid bridges are composed of liquid from both contacting particles. As an example: the liquid bridge volume in the upper left is composed of a fraction of liquid contents $L_{i}$ and $L_{j}$. Thus, the main part of the liquid content $L_{i}$ in Fig. $8 \mathrm{a}$ is still available for additional liquid bridges on particle $i$. By means of a geometrical tangent construction, the area of the spherical cap and hence, the contributing liquid contents $V_{k, i}$ and $V_{k, j}$ are determined according to Eq. (18):

$V_{k, \text { i or } j}=\frac{L_{\text {i or } j}}{2}\left(1-\sqrt{1-\frac{R_{\text {i or j }}^{2}}{\left(R_{i}+R_{j}\right)^{2}}}\right)$.

The sum of the two partial volumes yields the liquid bridge volume (Eq. 19) which is used to calculate the liquid bridge force:

$V=V_{k, i}+V_{k, j}$

The liquid bridge force is calculated as a function of separation distance as long as the rupture distance is not exceeded. When the rupture distance $S_{B}$ is reached, the liquid bridge will rupture and the force will disappear. In order to represent this behaviour in the DEM simulation, a contact radius has to be defined. This guarantees contact detection beyond (b)

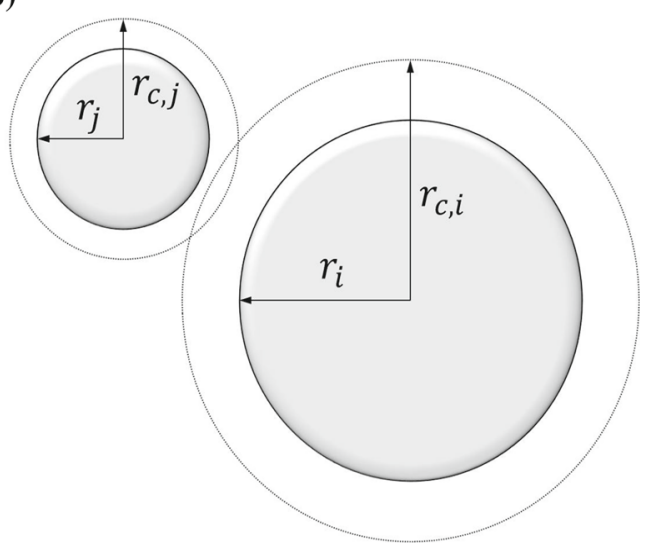

that is used for contact detection in the liquid bridge model. The liquid bridges would rupture after the physical contact if contact radius definition would be missing

physical contact (Hertz model). When selecting the contact radius, contact detection must be ensured as long as the rupture distance is not reached and no 'third' particle should slip into an existing liquid bridge. Accordingly, the contact radius should not be too small, but not too big either. In our case, a contact radius $r_{c} 1.3$ times larger than the physical radius is determined as an optimum (Fig. 8b). Since the liquid bridge force occurs simultaneously with the physical contact force and negative separation distances are not possible, the separation distance $S$ is set to zero when calculating the liquid bridge force during a physical contact.

After rupturing, the liquid volume is distributed according to the corresponding transfer ratio (see Eq. 17). Again, a case distinction is carried out. In the case of same sized particles with the same contact angles, the liquid will split in half $\left(T F_{i}=0.5\right)$. In the case of particle-wall collision, no liquid will be transferred, characterized by $T F_{i}=1$. The wall remains dry and the liquid returns to the previously contacting particle. Due to the proportional calculation of the liquid volume and the inclusion of the transfer ratio, liquid transportation in bulk materials can be simulated by the DEM.

In addition to liquid bridge forces in the normal direction, viscous forces arise from the presence of moisture. The relationship between viscous and capillary forces is characterized by the capillary number $C a$, which is calculated by Eq. (20), where $\eta$ is the dynamic viscosity and $u$ is a characteristic velocity. For the characteristic velocity, we used the maximum relative velocity in the normal direction $u_{r e l, \max }^{n}$ from the simulation of a dry mixing process at a rotational speed of $108 \mathrm{rpm}$ (see Fig. 2). As we only focus on water as a liquid, it is a reasonable assumption to neglect viscous forces (see Eq. 20) because of the very small capillary number. 
Fig. 9 Simulation of two wet mixing processes at $108 \mathrm{rpm}$ with different contact angles of the additive component. Total amount of added water $m_{l, \text { total }}=20.37 \mathrm{~g}$, surface tension $\gamma=0.073 \mathrm{~N} / \mathrm{m}$. a Mixing efficiency as a function of the dimensionless time $\left(t_{\text {Max }}=20 \mathrm{~s}\right)$. b 3D visualization of solid mixing process (corresponding to the dashed line $\theta_{j}=0^{\circ}$ ) (a)

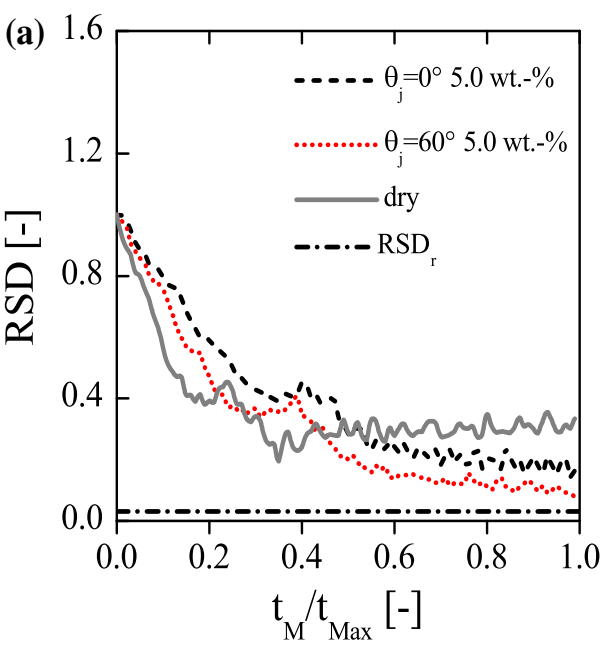

(b)

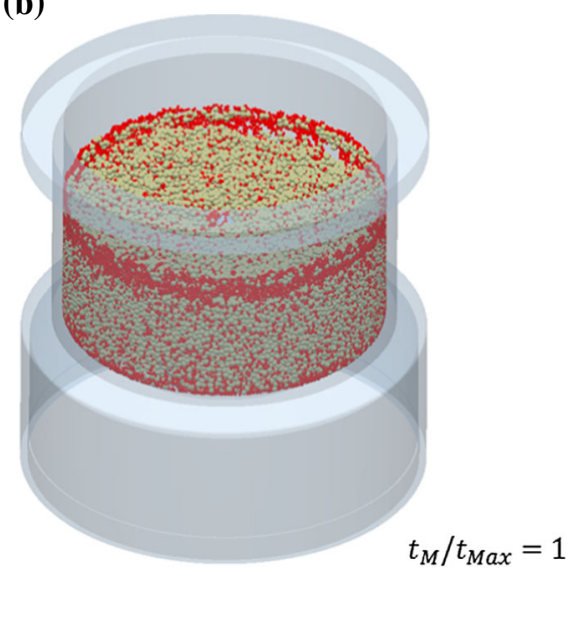

$$
\begin{aligned}
C a & =\frac{\eta u}{\gamma}=\frac{\eta u_{r e l, \max }^{n}}{\gamma} \\
& =\frac{0.001 \mathrm{Pas} \cdot 0.68 \mathrm{~m} / \mathrm{s}}{0.073 \mathrm{~N} / \mathrm{m}}=0.009
\end{aligned}
$$

We also neglected the gravitational force of the liquid. In the simulation shown in the following Sect. 4, the total amount of added water (5 wt $\%$ ) is set to $m_{l, \text { total }}=20.37 \mathrm{~g}$ which is quite low in comparison to the total amount of particulate solids $\left(m_{\text {solids }}=m_{\text {additive }}+m_{\text {filler }}=50.97 \mathrm{~g}+\right.$ $407.7 \mathrm{~g}=458.7 \mathrm{~g}$ ). If the gravitational force is related to the liquid bridge force, the modified 'liquid Bond number' $B o_{l}$ will reach a maximum magnitude of 0.003 . Hence, the liquid bridge force is at least 100 times larger than the force due to gravity (Eq. 21). The gravitational and viscous forces have already been discussed in the work of Remy et al. [12], who made a first DEM simulation of wet monodisperse spheres in a bladed mixer. Gravitational and viscous forces were neglected in their work as well.

$$
\begin{aligned}
B o_{l, \max } & =\frac{\text { gravitational force }}{\text { liquid bridge force }} \\
& =\frac{\text { gv } \rho_{f}}{2 \pi y_{\min } \gamma-\pi y_{\min }^{2} \Delta p}=0.003
\end{aligned}
$$

\section{Comparison of dry and wet mixing}

\subsection{Influence of the contact angle}

In the simulation of a wet mixing process the same initial conditions as shown in Fig. $1 \mathrm{~b}$ were chosen, with the difference being that we allocated a liquid content of $5 \mathrm{wt} \%$ of the particle mass to each gray filler particle. Accordingly, the total amount of liquid is perfectly distributed throughout the filler material in the beginning. The filler material has a contact angle of $\theta_{i}=0^{\circ}$. In this idealized system each gray particle is covered by a thin liquid layer, while the red additive component is dry, corresponding to $0 \mathrm{wt} \%$ of liquid. At this time $\left(t_{M} / t_{\text {Max }}=0\right)$, liquid bridges already exist between contacting filler particles. However, no liquid is transferred at this point of time. Virtual sampling and determining the mixing efficiency allow for a quantitative comparison between a dry and a wet mixing process (Fig. 9a).

The results of the dry mixing process were already shown in Fig. 2, where the uniform random mixture $R S D_{R}$ is not reached. Due to liquid addition, interparticle forces appear and reduce the relative particle mobility. It takes more time to distribute the additive particles in the cohesive filler material, which is indicated by the increase of mixing efficiencies. A smaller particle mobility than that seen for dry free-flowing material occurred in a tumbler mixer as well [26]. Compared to the dry mixing process, the final homogeneity of wet mixing is improved. This effect is similar to the reduction observed in segregation intensity for cohesive flows in a tumbling blender with a simplified cohesion model [27]. Even in wet mixing, however, the ideally mixed state cannot be achieved. This is due to the fact that a change of segregation mechanism occurs. While percolating segregation is observed during dry mixing, size-dependent heteroagglomerates are formed behind the blades of the mixing tool (Fig. 10) in the wet mixing process, resulting in a decrease of homogeneity. In this region low stress intensity is induced which causes suboptimal deagglomeration. In addition, an accumulation of the additive particle can be found at the vessel wall (Fig. 9b), which might be improved by a higher energy input or a conical vessel wall, as indicated in the work of Nakamura et al. [28].

Comparison of additive particles with different wetting properties reveals differences in the mixing process. Due to the smaller and shorter liquid bridge forces at a contact angle of $\theta_{j}=60^{\circ}$, the relative standard deviation decreases faster compared to the other wet mixing process (Fig. 9a). Hence, the final state is reached more quickly. Final homogeneity is 
(a)

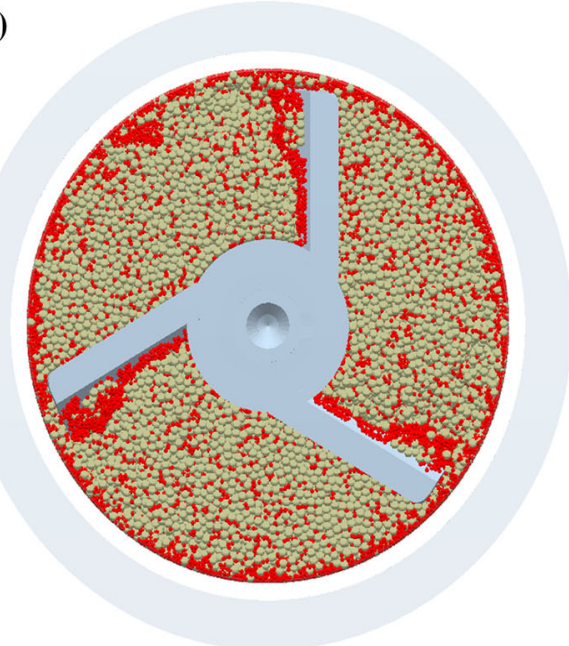

(b)

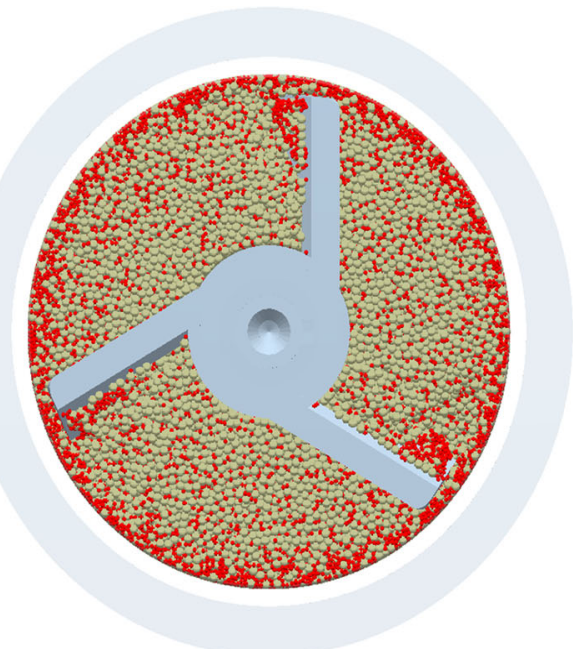

Fig. $103 \mathrm{D}$ Visualization of the bottom of the mixing vessel at the end of mixing $\left(t_{M} / t_{\text {Max }}=1\right)$ that show heteroagglomerates behind the blades of the mixing tool. $\mathbf{a} \theta_{j}=0^{\circ}$ (corresponding to the dashed line in Fig. 9a). $\mathbf{b} \theta_{j}=60^{\circ}$ (corresponding to the dotted line in Fig. 9a)

Fig. 11 Simulation of two wet mixing processes at $108 \mathrm{rpm}$ with different contact angles of the additive component. Total amount of added water $m_{l, t o t a l}=20.37 \mathrm{~g}$, surface tension $\gamma=0.073 \mathrm{~N} / \mathrm{m}$. (a): Illustration of liquid distribution as a function of the dimensionless time. b 3D visualization of the liquid distribution (corresponding to the dashed line $\theta_{j}=0^{\circ}$ )

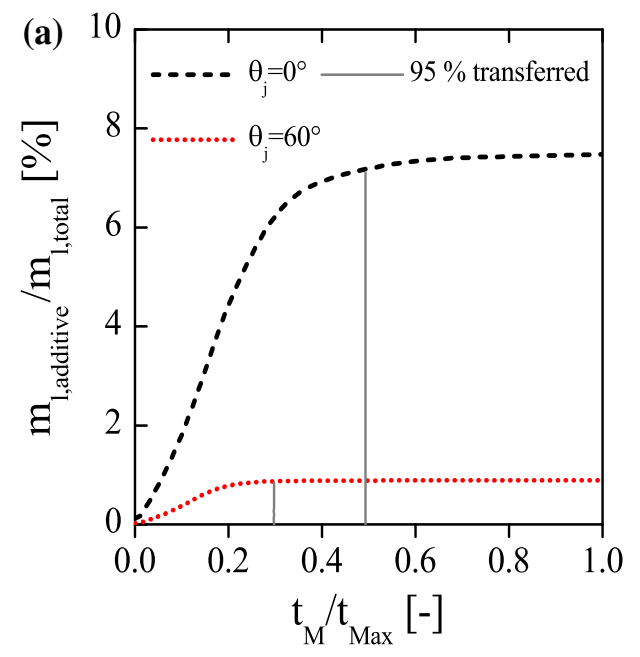

(b)

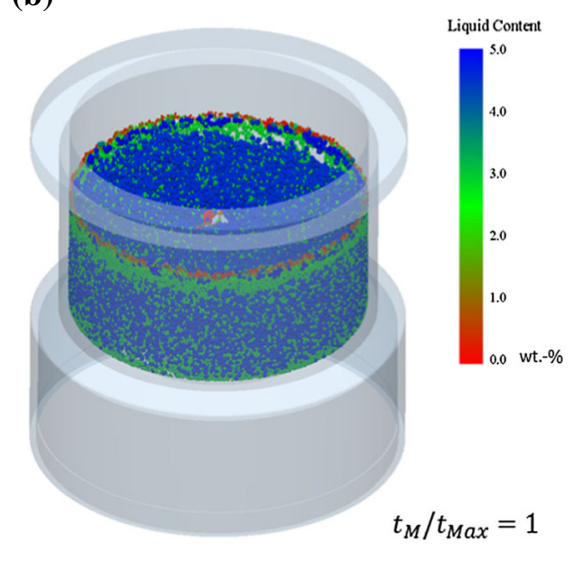

better compared to a low contact angle. Percolating segregation is not completely compensated by the addition of liquid. Accumulation of additive particles can still be observed in the gap between the mixing tool and the mixing vessel. This mechanism is a more pronounced at a high contact angle (Fig. 10b). As a result of the different liquid bridge forces, rupture distances and transfer ratios, less heteroagglomerates consisting of additive particles are observed at a high contact angle.

\subsection{Liquid transfer}

The distribution of liquid is shown in Fig. 11a. In the beginning of mixing the wet filler particles are colored blue, indicating a liquid content of $5 \mathrm{wt} \%$, while the dry additive particles are colored red. With progressing mixing time, the additive particles change their color from red to green. The blue filler particles lose some liquid, which is shown by the turquoise color. Only a small part of the total liquid content is transferred to the additive particles, while most of the liquid remains in the filler material. This is explained by the fact that smaller particles can carry less liquid than larger particles. Figure 11a shows the speed of liquid distribution within the additive component. At a low contact angle, approximately $7 \%$ of the total liquid is transferred to the additive component. Only approximately $1 \%$ is transferred when a high contact angle is chosen. The reduced liquid adsorption by the smaller particles in the case of a high contact angle is a logical, physical result. Poor wetting properties mean less affinity to water. In the case of good wetting properties, more collisions are necessary to transfer the liquid. Hence, the equilibrium state is reached quicker, when a high contact angle is used. This observation, in turn, underlines the slightly faster mixing efficiency at a contact angle of $60^{\circ}$ in Fig. 9a.

The reason why a steady-state of uniformly distributed liquid will be reached at the end of mixing is based on the spherical cap model (see Fig. 8). Several collisions are necessary to achieve a complete liquid exchange process [24]. At steady-state, the liquid contributing to a newly created liq- 
Fig. 12 Mixing efficiency as a function of the dimensionless time $\left(t_{\mathrm{Max}}=20 \mathrm{~s}\right)$, surface tension $\gamma=0.073 \mathrm{~N} / \mathrm{m}$. a Simulation of three wet mixing processes at $108 \mathrm{rpm}$ with different contact angles between the filler and the additive component in order to investigate the influence of liquid content. b Simulation of six wet mixing processes with $5 \mathrm{wt} \%$ of liquid at different rotational speed
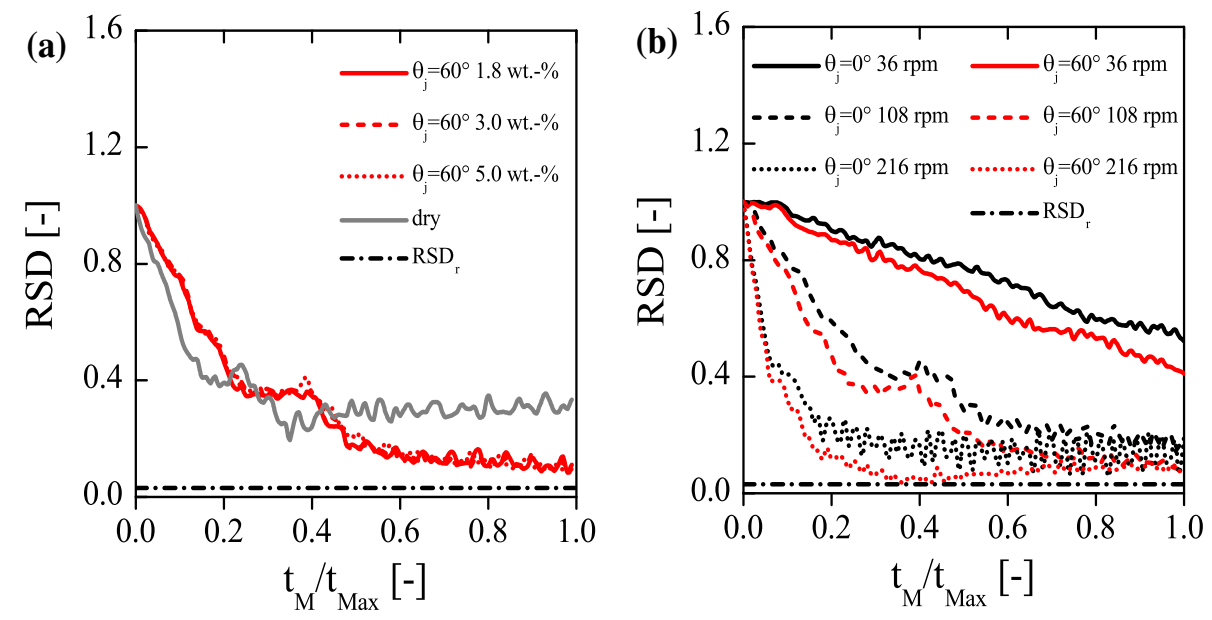

uid bridge is exactly the same as the liquid transferred after rupturing. Hence, no further liquid exchange takes place, resulting in homogeneously distributed liquid at the end of mixing.

When liquid is drawn and dispersed between the particles, a uniform liquid distribution seems logical at first glance due to wetting, agglomeration, breakage and liquid migration. However, Mani et al. [29] observed a depletion of liquid in shear bands with uniform velocity gradient and proposed a new diffusive liquid dynamics model depending on the local shear rate [30]. If those flow dynamics were taken into account in this work, a fluctuation around the mean stationary end value in Fig. 11 would most likely be feasible. Additionally, it is a rough assumption in our model to distribute the liquid as a thin film on a poor wetting additive material. For this reason, partial wetting of the particle surface [21] and droplet modelling has to be taken into account in ongoing work. Then, experimentally observed selective enrichment of liquid in the wetting filler component [8] might be observable in numerical simulation as well.

\subsection{Influence of liquid content and rotational speed}

Simulations were performed using different amounts of liquid and different rotational speeds. The variation of different liquid contents shows an almost undetectable effect (Fig. 12a). From a mathematically point of view this is due to the small volume dependency of the liquid bridge force at small particle distances as shown in Fig. 5 and discussed by Butt and Kappl [15]. Hence, the volume-dependent rupture distance has a smaller influence on the dispersive particle mobility than the contact-angle-dependent liquid bridge force. As a result, the size of the agglomerates is hardly changed with the amounts of liquid. This effect has already been observed with small amounts of liquid in a bladed mixer [12]. However, we cannot observe a liquid content dependent optimum $[12,26]$ which may be explained due to the different mixing principles, different geometries and higher shear forces. This observation, however, is limited due to the assumption of the pendular liquid bridges in this work. It still remains questionable how the mixing efficiency is influenced when moving into the funicular regime at higher liquid contents.

The effect of blade rotation speed on the mixing efficiency was investigated computationally at three different speeds: 36, 108 and $216 \mathrm{rpm}$, respectively. A direct correlation between the dispersive particle mobility and the rotational speed can be observed in Fig. 12b. Due to higher shear forces smaller agglomerates are formed which are more mobile than bigger ones. This results in an enhancement of mixing kinetics indicated by the fast decrease of the RSD. The effect of breaking up more coherent particle bonds is observed at higher tumbler speeds as well [27]. There is little energy input at $36 \mathrm{rpm}$, which is not sufficient to reach the final homogeneity after a simulation time $t_{\max }$ of $20 \mathrm{~s}$. The speed increase up to $216 \mathrm{rpm}$ results in a better end homogeneity compared to $108 \mathrm{rpm}$, explained by less agglomerate formation at the mixing tool blades. Comparing different contact angles of the additive component, a faster decrease of the RSD can be observed for larger contact angles at the three different rotational speeds. This smaller dispersive particle mobility results from the slightly lower cohesive forces at $60^{\circ}$.

\section{Conclusion}

We present new empirical functions for the liquid bridge force, the transfer ratio, and the rupture distance by solving the Young-Laplace equation numerically. These equations can be used for a liquid contact event in the DEM simulation of different materials, but are limited to a particle size ratio of two and $\theta_{i}=0^{\circ}$. Using the new model equations, it is possible to simulate mixing processes of dry and wet particles. Quantitative comparison of a completely dry and partly wet mixing processes reveal an improvement in homogene- 
ity when a small amount of liquid is added to the mixing components. Due to interparticle liquid bridge forces, percolating segregation is reduced. Because of the size difference between the filler and the additive component, heteroagglomeration occurs at the mixing tool blades. This change in segregation mechanism reduces homogeneity. Accordingly, the best technically possible homogeneity cannot be achieved even in moist mixing processes. Differences are observed when varying the contact angle of the additive component. More heteroagglomerates behind the mixing tool occur in the case of a small contact angle of the additive component. While the total amount of liquid virtually has no influence on the degree of mixing, the particle mobility increases with rotational speed. Dynamic liquid migration, partial wetting of the particle surface and droplet modelling might improve the liquid distribution model in ongoing work.

Acknowledgements The authors gratefully acknowledge the financial support by the Deutsche Forschungsgemeinschaft (DFG NI 414/25-1).

\section{References}

1. Johanson, J.R.: Particle segregation... and what to do about it. Chem. Eng. 85(11), 183-188 (1978)

2. Williams, J.C.: The segregation of particulate materials. A review. Powder Technol. 15(2), 245-251 (1976). https://doi.org/10.1016/ 0032-5910(76)80053-8

3. Bridgwater, J.: Fundamental powder mixing mechanisms. Powder Technol. 15(2), 215-236 (1976)

4. Marinelli, J., Carson, J.W.: Solve solids flow problems in bins, hoppers, and feeders. Chem. Eng. Prog. 88(5), 22-28 (1992)

5. Gögelein, C., Brinkmann, M., Schröter, M., Herminghaus, S.: Controlling the formation of capillary bridges in binary liquid mixtures. Langmuir 26(22), 17184-17189 (2010)

6. Lian, G., Seville, J.: The capillary bridge between two spheres: New closed-form equations in a two century old problem. Adv. Colloid Interface Sci. 227, 53-62 (2016). https://doi.org/10.1016/ j.cis.2015.11.003

7. Johanson, J.R.: Smooth out solids blending problems. Chem. Eng. Prog. 96(4), 21-37 (2000)

8. Hoffmann, T.: Mischen und Befeuchten von Schuttgutern. Universität-Gesamthochschule-Paderborn (1995)

9. Cundall, P.A., Strack, O.D.: A discrete numerical model for granular assemblies. Geotechnique 29(1), 47-65 (1979)

10. Tsuji, Y., Tanaka, T., Ishida, T.: Lagrangian numerical simulation of plug flow of cohesionless particles in a horizontal pipe. Powder Technol. 71(3), 239-250 (1992). https://doi.org/10.1016/00325910(92)88030-L

11. Mikami, T., Kamiya, H., Horio, M.: Numerical simulation of cohesive powder behavior in a fluidized bed. Chem. Eng. Sci. 53(10), 1927-1940 (1998). https://doi.org/10.1016/S00092509(97)00325-4

12. Remy, B., Khinast, J.G., Glasser, B.J.: Wet granular flows in a bladed mixer: experiments and simulations of monodisperse spheres. AIChE J. 58(11), 3354-3369 (2012)
13. Soulie, F., Cherblanc, F., El Youssoufi, M.S., Saix, C.: Influence of liquid bridges on the mechanical behaviour of polydisperse granular materials. Int. J. Numer. Anal. Methods Geomech. 30(3), 213-228 (2006)

14. Anand, A., Curtis, J.S., Wassgren, C.R., Hancock, B.C., Ketterhagen, W.R.: Segregation of cohesive granular materials during discharge from a rectangular hopper. Granul. Matter 12(2), 193$200(2010)$

15. Butt, H.-J., Kappl, M.: Normal capillary forces. Adv. Colloid Interface Sci. 146(1), 48-60 (2009)

16. Derjaguin, B.: Untersuchungen über die Reibung und Adhäsion, IV. Kolloid-Zeitschrift 69(2), 155-164 (1934). https://doi.org/10. 1007/bf01433225

17. Alchikh-Sulaiman, B., Ein-Mozaffari, F., Lohi, A.: Evaluation of poly-disperse solid particles mixing in a slant cone mixer using discrete element method. Chem. Eng. Res. Des. 96, 196-213 (2015). https://doi.org/10.1016/j.cherd.2015.02.020

18. Stieß, M., Ripperger, S.: Mechanische VerfahrenstechnikPartikeltechnologie 1, vol. 1. Springer, Berlin (2009)

19. Fisher, R.A.: On the capillary forces in an ideal soil; correction of formulae given by W. B. Haines. J. Agric. Sci. 16(03), 492-505 (1926). https://doi.org/10.1017/S0021859600007838

20. Hotta, K., Takeda, K., Iinoya, K.: The capillary binding force of a liquid bridge. Powder Technol. 10(4-5), 231-242 (1974). https:// doi.org/10.1016/0032-5910(74)85047-3

21. Schubert, H.: Kapillarität in porösen Feststoffsystemen. Springer, Berlin (1982)

22. Lian, G., Thornton, C., Adams, M.J.: A theoretical study of the liquid bridge forces between two rigid spherical bodies. J. Colloid Interface Sci. 161(1), 138-147 (1993). https://doi.org/10.1006/jcis. 1993.1452

23. Willett, C.D., Adams, M.J., Johnson, S.A., Seville, J.P.: Capillary bridges between two spherical bodies. Langmuir 16(24), 93969405 (2000)

24. Shi, D., McCarthy, J.J.: Numerical simulation of liquid transfer between particles. Powder Technol. 184(1), 64-75 (2008). https:// doi.org/10.1016/j.powtec.2007.08.011

25. Pitois, O., Moucheront, P., Chateau, X.: Liquid bridge between two moving spheres: an experimental study of viscosity effects. J. Colloid Interface Sci. 231(1), 26-31 (2000)

26. McCarthy, J.J.: Micro-modeling of cohesive mixing processes. Powder Technol. 138(1), 63-67 (2003). https://doi.org/10.1016/ j.powtec.2003.08.042

27. Chaudhuri, B., Mehrotra, A., Muzzio, F.J., Tomassone, M.S.: Cohesive effects in powder mixing in a tumbling blender. Powder Technol. 165(2), 105-114 (2006). https://doi.org/10.1016/j. powtec.2006.04.001

28. Nakamura, H., Fujii, H., Watano, S.: Scale-up of high shear mixergranulator based on discrete element analysis. Powder Technol. 236, 149-156 (2013). https://doi.org/10.1016/j.powtec.2012.03. 009

29. Mani, R., Kadau, D., Or, D., Herrmann, H.J.: Fluid depletion in shear bands. Phys. Rev. Lett. 109(24), 248001 (2012)

30. Mani, R., Kadau, D., Herrmann, H.J.: Liquid migration in sheared unsaturated granular media. Granul. Matter 15(4), 447-454 (2013). https://doi.org/10.1007/s10035-012-0387-3 


\section{Repository KITopen}

Dies ist ein Postprint/begutachtetes Manuskript.

Empfohlene Zitierung:

Schmelzle, S.; Nirschl, H.

DEM simulations : mixing of dry and wet granular material with different contact angles. 2018. Granular matter, 20.

doi: $10.5445 / / R / 1000081175$

Zitierung der Originalveröffentlichung:

Schmelzle, S.; Nirschl, H.

DEM simulations : mixing of dry and wet granular material with different contact angles. 2018. Granular matter, 20 (2), Art.Nr. 19.

doi:10.1007/s10035-018-0792-3 